\title{
Patient management positive covid: the central role of clinical nutrition and supplementation of essential amino acids during and after hospitalization..
}

\author{
Francesco Ferrara ${ }^{1}$, Giovanni Granata ${ }^{2}$, Chiara Pelliccia ${ }^{3}$, Raffaele La Porta ${ }^{4}$, and Antonio \\ Vitiello ${ }^{1}$ \\ ${ }^{1}$ Azienda Unità Sanitaria Locale Umbria 1 \\ ${ }^{2}$ Local Health Authority Salerno \\ ${ }^{3}$ Azienda Unità Sanitaria Locale Umbria 2 \\ ${ }^{4}$ ASUR Marche
}

April 27, 2020

\begin{abstract}
Background The covid 19 positive patient who is subject to a hyperinflammatory condition associated with lung injury with the development of pneumonia is hospitalized in the intensive care unit. Before resolving and overcoming the "cytokine storm", with overexpression of pro-inflammatory interleukins (IL-, Il-6), this patient will be intubated for more than 48 hours and therefore needs adequate nutrition. Experimental approach Malnutrition can lead to sarcopenia with a decrease in lean body mass and worsening of the inflammatory state underway. In addition, severe debilitation, if not corrected with adequate nutrition, can greatly lengthen rehabilitation times with prolonged hospitalization, increased costs and reduced turn over already in crisis due to the health emergency caused by coronavirus. Key Results The aim of this study is to focus attention on the nutritional importance that must be provided in case of covid 19 together with pharmacological treatments to lower the number of circulating proinflammatory cytokines. Conclusions Oral, enteral and parenteral nutrition should always be carried out according to the patient's condition and, in the case of a hyperinflammatory patient, such as the one affected by covid 19, it has been shown that the supplementation of amino acids helps to lower the inflammatory state and promotes normal recovery physiological. Keywords: amino acids, nutrition, covid, glutamine, hyperinflammatory, sarcopenia, cytokine.
\end{abstract}

\section{Introduction}

A newly identified coronavirus (SARS-CoV-2) in China in December 2019 resulted in a significant number of pneumonia resulting in deaths. The spread, as usual of these viruses, is rapidly spreading all over the world with thousands of thousands of deaths. It is no coincidence that the WHO has declared a state of pandemic. For these reasons, worldwide attention is concentrated in the fight against this virus.

The genomic sequence of SARS-CoV-2 is $79.5 \%$ of the identity with SARS-CoV. The evidence that caused Cov2 sars infection causes a generalized hyper inflammatory state due to the release of cytokines ("cytokine storm").

In literature it has been shown that the final stage of infection is the deadly one for humans. In fact, it is not the viral phase that kills but the strong activation of the immune system and the consequent release of cytokines. The patient is thus supported and hospitalized in an intensive care unit (ICU) hoping that he will overcome the "famous storm" and remain alive.

World scientific societies, health professionals, doctors, researchers and scholars are intensifying research to 
find a suitable cure to fight the virus, both to prevent infection and to treat the patient before he arrives at death. The vaccine will only be available in several months and at present many drugs are being examined without great success. The latest works give hope about colchicine and hydroxychloroquine/chloroquine also used in combination in order to avoid the release of cytokines with excellent chances of success.

The spontaneous reflection is the possibility of resorting to clinical nutrition and amino acid supplementation both to prevent, support and overcome the infectious phase with greater ease also in combination with pharmacological treatments.

This idea is reinforced by the fact that clinical nutrition and amino acids, in addition to adequately nourishing the patient bedridden in ICU, can modulate the immune system to prevent infection and ensure that the inflammatory state is kept under control with less release of cytokines. Above all, amino acids seem fundamental as an additional contribution in the patient suffering from covid and hospitalized in ICU: on the one hand it mitigates the release of cytokines and on the other it favors a prompt de-hospitalization in the healing phase for a prompt turn-over in the pandemic phase and with consequent savings of indirect and intangible costs (1-2).

\section{Hospitalization and nutrition in a positive Covid patient}

COVID-19 disease, caused by SARS-CoV-2 virus infection, can lead to manifestations even severe clinics such as pneumonia with respiratory failure, respiratory distress syndrome acute (ARDS), sepsis and septic shock, which require urgent hospitalization in ICU. ARDS in particular manifests itself as a serious failure hypoxic respiratory accompanied by inflammation, pulmonary edema and risk of dysfunction multi-organ and is often required mechanical ventilation invasive. Generally patients who died from SARS-CoV-2 infection they have an average age of 78 years and 3 or more comorbidities including chronic non-pathologies transmissible diseases such as ischemic heart disease, hypertension, diabetes and chronic obstructive pulmonary disease (COPD).

This is why COVID-19 is the new $\beta$-coronavirus, which can lead to various pulmonary phenomena and death $(\sim 10 \%)$ and represents a global health challenge. Medical nutrition therapy is among the pillars of therapeutic principles and a fundamental tool among global therapeutic measures for patients with COVID19.

Malnourished people with low immunity and chronic diseases have a worse higher prognosis and mortality rates. The relevance of nutritional therapy is important and should be implemented as a first-line treatment and implemented in the anti-Covid-19 clinical practice standard. Good nutrition not only provides the body with immunity to disease, including COVID-19, but it is also the main guarantee for the promotion of the recovery of the disease and the infected patient. However, the indications for nutritional therapy must be tailored. Nutritionists should pay attention to the most appropriate artificial nutrition (AN) approach with enteral nutrition (EN) or parenteral nutrition (PN). Plan, the method, route and formula of nutritional therapy must be adapted dynamically and promptly in accordance with the clinical characteristics of the patient COVID-19. The transition between ONS, EN and PN should be fluid, following the principle that when the EN can satisfy $50 \%$ of the target demand, the PN can be constantly reduced and carried out arrested; when the ONS can satisfy $50 \%$ of the target demand, the EN it can be progressively reduced and therefore arrested. 


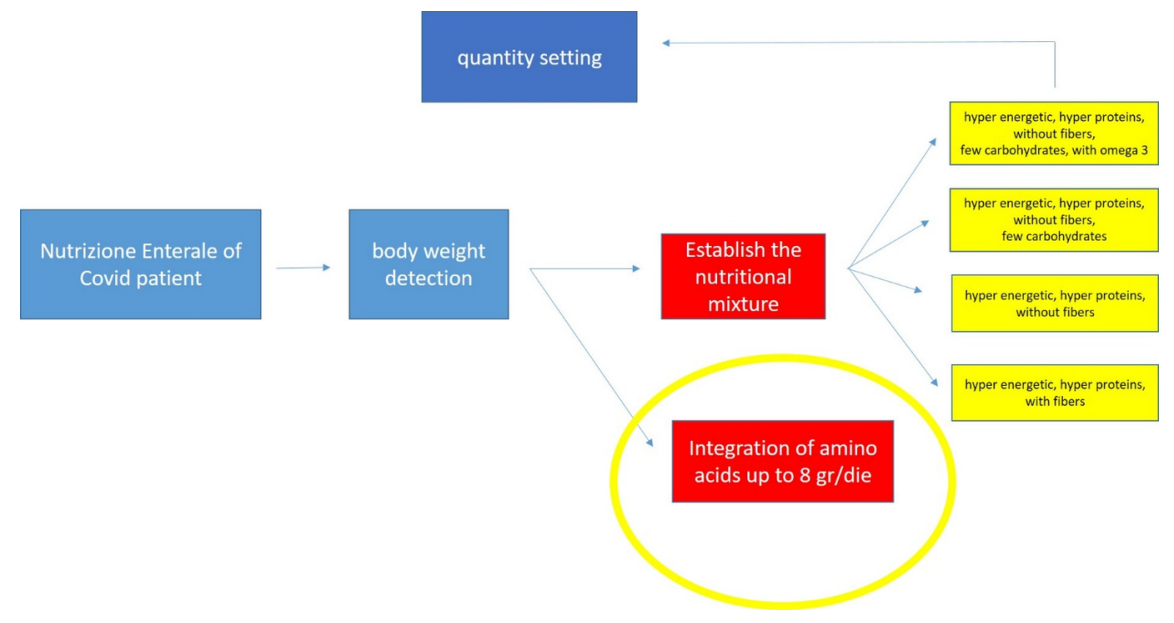

Figure 1: Enteral nutritional support in the positive covid patient.

Advanced age and comorbidity is related to fragility, a syndrome characterized by reduction of functional reserves and decreased resistance to stress. Fragility is connected with loss weight and malnutrition and acute hospitalization without adequate nutritional support can worsen one situation already compromised. All patients hospitalized for more than 48 hours, regardless of the index of initial body mass (BMI) and age, are at risk of malnutrition and need timely and appropriate nutritional intervention. Prolonged hospitalization following catabolism and immobilization, induces a decrease in weight and muscle mass that can result in sarcopenia, condition which in turn helps to reduce respiratory and cardiac function, prolong the hospitalization of the patient and worse prognosis. So counter or prevent malnutrition hospital by improving the patient's response to therapy and facilitating healthcare professionals in the hospital nutrition management by reducing the already high workload due to the state of emergency.

Most patients have severe inflammation and anorexia which leads to one drastic reduction of food intake which then leads to a respiratory failure treated with continuous positive airway pressure. High calorie diets must be readily provided. Oral integration of whey protein and intravenous infusion of multivitamin and multimineral solutions must be implemented at the time of admission. If respiratory conditions worsen, total parenteral nutrition should be considered. To cope with the current emergency crisis, nutritional assistance must be implemented promptly and pragmatically in patients with COVID-19 because it could be neglected although it is necessary for the benefit of clinical and effective results in preventing the consequences of malnutrition in this patient population. 


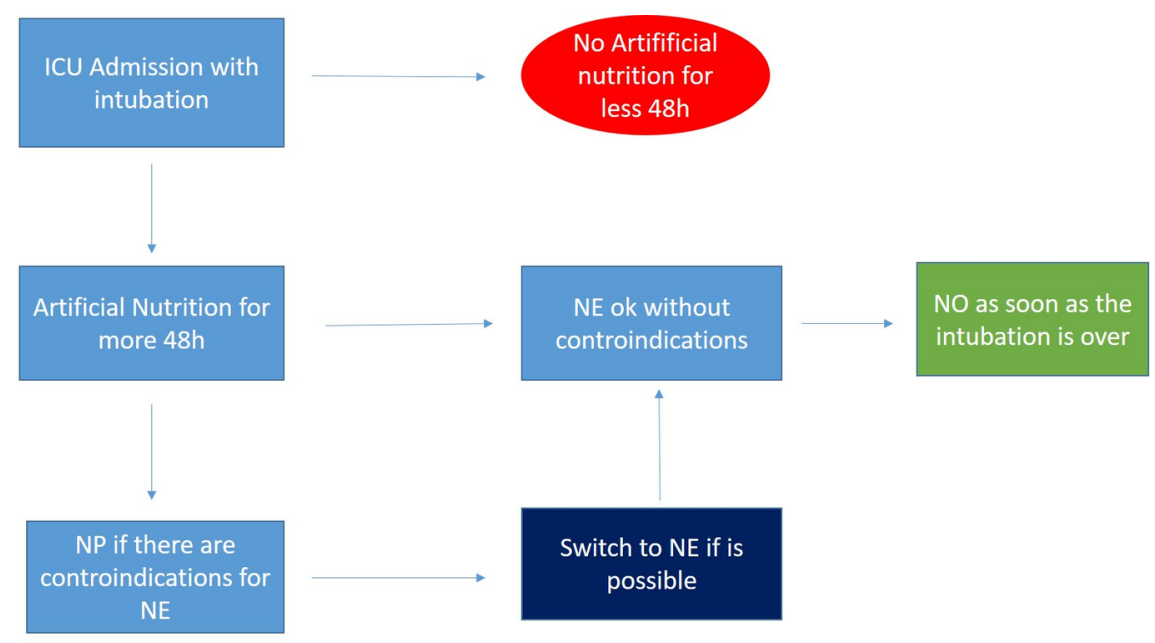

Figure 2: Oral, enteral or parenteral nutrition depending on clinical conditions.

It is possible to follow simple patterns of conduct. In the critical patient in intensive care with respiratory failure for over 48 hours EN medical nutritional therapy must be started with priority; PN nutrition has been started for more than 3 days after EN strategies have failed to avoid severe malnutrition. Overeating should always be avoided and a gradual approach is best. The nutritional requirement in COVID-19 must provide more energy than normal nutrition. Maintaining the energy balance of patients with COVID-19 is fundamental. However, considering the increased metabolic load in patients with severe pneumonia, moderately low calories can reduce the metabolic load and excessive energy intake is a risk factor. To reduce the catabolism due to inflammatory mediators, it is indicated to increase protein intake as a top priority. The supply of amino acids helps prevent muscle loss and improve the strength of the respiratory muscles.

Given the importance clinical, ethical and economic action aimed at reducing infections, including those from Covid-19, studies have always been carried out which demonstrate how the correct supply of nutrients can improve the prognosis and pathological course in ICUs. The use of amino acids undoubtedly it improves the clinical course and favors the prompt physiological recovery after a period of hospitalization. Then, in the case of the covid patient, admitted to ICU with a strong inflammatory state, it is shown how the adequate nutritional support of glutamine, probiotics and lactoferrin helps to reduce the pro-inflammatory molecules (IL-1, IL-6) responsible for the fatal event pulmonary coronavirus.

All the figures of professionals and health workers, engaged in the delicate task of facing and stemming the serious phenomenon, should be more sensitized on the issue of nutrition-immunological ability, with reference to both physiology and general pathology and disease specifications in particular as in the case of Sars-Cov-2.

Alterations in muscle protein turnover, if the patient remains hospitalized for a long time, could lead to physical disability. Hypercatabolic activity if integrated with amino acids could limit muscle hypercatabatabism (MH) (3-9).

\section{Supplementation of amino acids in the hyperinflammatory state}

Inflammation and changes in amino acid metabolism are related to Covid e affect the skeletal muscles, therefore probably rehabilitation requires personalized approaches. In addition amino acids they are essential nutrients for infectious microorganisms and the levels of some amino acids such as tryptophan decrease in response to infection and / or inflammation and for this reason they should be restored so as not to experience severe malnutrition.

Some nutrients, including amino acids, have been shown to have a remarkable amountinfluence on immune function (immunonutrients) and improvement of metabolic and nutritional indices, such as nitrogen balance 
and serum proteins. Immunonutrients can promote patient recovery by inhibiting inflammatory responses and regulating immune function. Glutamine which can modulate and preserve intestinal and lung function, compromised in conditions of severe stress, reducing the production of pro-inflammatory cytokines. Cytokine mediated effects are an essential part response to infection but excessive production of pro-inflammatory cytokines increases the risk of pneumonia and death in Covid-19. The production of pro-inflammatory

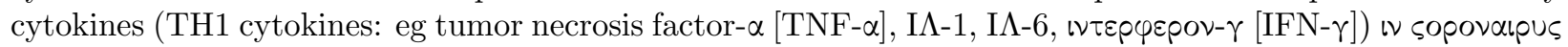

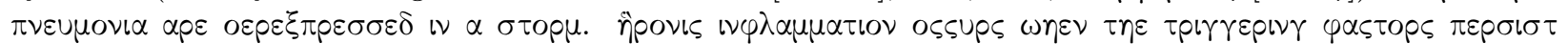

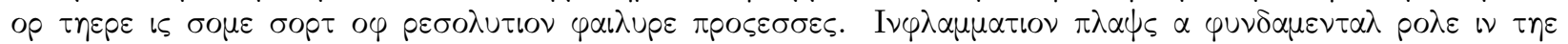

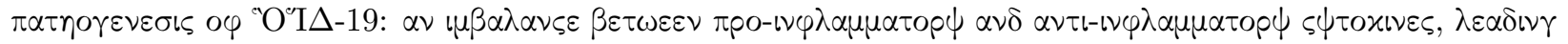

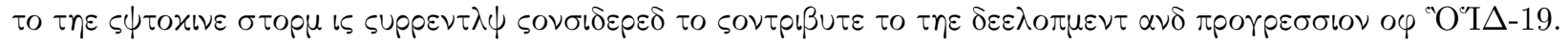

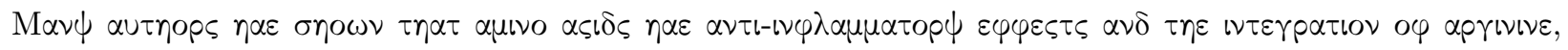
$\gamma \lambda \cup \tau \alpha \mu i v \varepsilon$ op $\gamma \lambda \psi s i v \varepsilon$ น

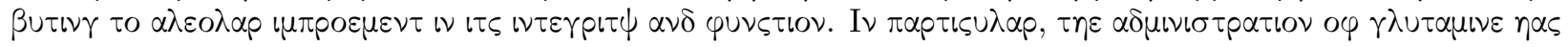

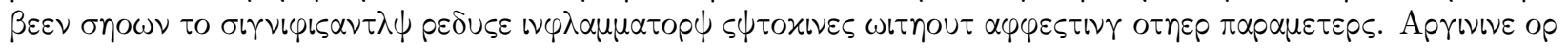

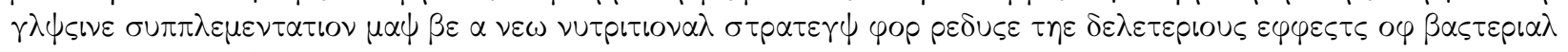

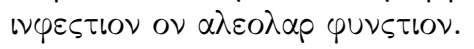

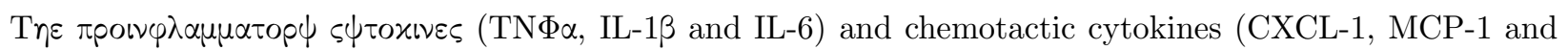
ICAM-1) produced by immune cells are powerful inducers of apoptosis in alveolar epithelial cells and cause a severe inflammatory state and the use of amino acids has reduced mRNA levels for TNF $\alpha$, IL- $1 \beta$ and IL- 6 indicating a reduction inflammatory response. Importantly, IL-6 promotes expression of CXCL-1, which in turn regulates neutrophils accumulation and contributes to lung injury. The administration of arginine or glycine is associated with reduced expression of IL-6, CXCL-1 and decrease in the accumulation of neutrophils in the alveolar epithelium, indicating a regulatory effect of chemokine secretion in lung tissues. Whereas the inhibition of CXCL-1 reduces the accumulation of neutrophils in the lungs tissues and protects against lung injury in clinical patients, arginine or glycine can be used as a new adjuvant nutrient in clinical therapy. Further studies are needed to clarify molecular mechanisms underlying this important modulatory effect of arginine, glycine and glutamine.

Glutamine inhibits the accumulation of white blood cells and Arginine, glycine or glutamine have been reported to inhibit cell death in various studies but the metabolism of amino acids and the production of their metabolites differ from each other in alveolar epithelial cells, thus leading to different responses.

Furthermore, it should be added that malnutrition easily leads to a decrease in the lean body mass and can cause sarcopenia, a frequently encountered disease in the elderly with aggravation of geriatric pathologies. Current opinions consider malnutrition and possible sarcopenia as a consequence of multiple medical, behavioral and environmental factors involving the nervous, hormonal, nutritional system, substitution of muscle proteins, proinflammatory conditions and the load of reactive oxygen species inside of mitochondria. With the intake of amino acids there is a better functional recovery of the muscles and a significant reduction in serum cancer necrosis factor- (TNF-) and a significant increase in both insulin-like growth factor without having significant adverse effects. These preliminary data indicate that dietary supplements with the oral amino acid mixture have significantly increased lean body mass in elderly subjects with and without sarcopenia (10-15).

\section{The role of glutamine}

Glutamine is one of the most abundant amino acid in the human organism and it is mostly present in the muscle tissue (about 60\%). It is particularly involved in a variety of processes for maintaining body homeostasis: redox processes, acid-base balance and glucose metabolism. Glutamine can become essential and have a positive effect in critically ill patients (e.g. in hospital stay). The effect of Glutamine seems to depend on the route of administration for which the parental one is proven to be the most effective so far. An excessive inflammatory response, oxidative stress, comorbidity such as malnutrition and a catabolic state, could lead to a severe depletion of glutamine. Respiratory diseases are mostly associated with a glutamine in- 
crease. Unlike skeletal muscle, which contains significant quantities of free glutamine, the lung synthesizes it ex novo from glutamate and ammonia by means of the enzyme glutamine-synthetase. The release of glutamine in stressed lungs is a consequence of the release of glucocorticoids and other mechanical mechanisms and becomes significant due to the massive pulmonary perfusion. Glutamine is synthesized from cytosolic glutamine synthetase (GS) in many tissues, but degraded by mitochondrial glutaminase (GA) and used in large quantities by other tissues that do not synthesize it. The beneficial effects of glutamine on critical diseases is thought to depend on a better expression of thermal shock proteins (HSP). In critically ill patients, the increase in mortality was associated with a decrease in plasma Gln concentration. It is proven that during catabolic stress, the consumption rate of Gln exceeds the supply and both the plasma pools and the skeletal muscle pools present severely reduced free Gln levels. Experimental studies have reported that Gln can protect cells, tissues and whole organisms from stress and injury through the following mechanisms: attenuation of activation of NF (nuclear factor) $-\varkappa \mathrm{B}$, a balance between pro and anti-inflammatory cytokines, reduction of neutrophils accumulation, improvement of intestinal integrity and immune cell function and enhancement of heat shock protein sex pression. In conclusion, high doses of parenteral Gln (>0.50 g / kg / day) show a greater benefit potential in critically ill patients, although the pathophysiological mechanisms of Gln require clarification (16-17).

\section{The role of lactoferrin}

Lactoferrin is a highly conserved natural pleiotropic glycoprotein of $80 \mathrm{kDa}$ and 703 amino acidic residues, it binds iron and is expressed and secreted by glandular cells. It can be found in most body fluids the molecule is equipped with in vitro antiviral properties against a wide range of viruses including SARS CoV, a virus belonging to the coronavirus family and naturally related to SARS CoV2, responsible for the current Covid19 pandemic. It presents immune-modulating and anti-inflammatory characteristics that can modify the host's responses to infections, thus it can be useful in association with other drugs in the most severe cases. Isolated for the first time in cow's milk and then in breast milk, it is used as a nutritional additive and if administered in doses between 4.5 and $100 \mathrm{mg}$ per day, is free of toxicity. There are also liposomal formulations. The antiviral activity of Lf has been demonstrated for the first time in infected mice where it induced polycythemia of the Friend virus complex in 1980. It attacks naked and enveloped viruses, both DNA and RNA, such as cytomegalovirus, herpes simplex virus, human immunodeficiency virus (HIV), rotavirus, poliovirus, respiratory syncytial virus, hepatitis $\mathrm{B}$ and $\mathrm{C}$ virus (HCV) parainfluenza virus, alphavirus, hantavirus, human papillomavirus, adenovirus, enterovirus 71, echovirus 6, influenza A virus and the Japanese encephalitis virus, with EC 50 (actual concentration) in vitro of Lf generally numbered in the micro-molar when against viruses. The ability of Lf to inhibit viral entry can be explained by the binding with cell's surface molecules (proteoglycans of heparan sulfate, HSPG) or viral particles (see HCV) or both. In addition to preventing the access of the virus, it can contribute by blocking the replication mechanisms of the virus once it enters. The theory according to which children between the ages of 0 and 10, therefore breastfeeding or using milk derivatives containing this molecule, have a reduced incidence of contracting the virus has been demonstrated, although it still needs to be validated. Furthermore, zinc saturated lactoferrin can be used in anti-inflammatory and preventive therapy against Covid19 (18).

\section{Conclusions}

The suggestions for proper evidence-based nutritional therapy reported here are for patients with critical COVID-19 conditions and should be included in life-saving therapies. The supplementation of amino acids and lactoferrin should always be accompanied by drug therapies following an ICU hospitalization and its importance cannot be overlooked. Malnutrition phenomena weaken the patient already in poor condition and greatly lengthen recovery and recovery times. Furthermore, the lung damage caused by oronavirus is caused by an overexpression of proinflammatory molecules (cytokines) which, from literature data, has been shown to be lowered by an adequate integration of amino acids. Nutritional therapy together with pharmacological therapy undoubtedly helps the covid patient to overcome the acute phase of the disease first and to shorten recovery times.

\section{MAIN STATEMENTS}


I, the undersigned, Francesco Ferrara and any other author, declare that:

- We have no conflict of interest;

- We have not received funding;

- There are no sensitive data and no patients were recruited for this study;

- The document does not conflict with ethical legislation.

Regards

The authors

\section{References}

1. Riccardo Caccialanza M.D et al. Early nutritional supplementation in non-critically ill patients hospitalized for the 2019 novel coronavirus disease (COVID-19 Rationale and feasibility of a shared pragmatic protocol. Nutrition 000 (2020) 110835.

2. L. ROMANO et al. Short Report-Medical nutrition therapy for critically ill patients with COVID-19. Eur opean Review for Medical and Pharmacological Sciences 2020; 24: 4035-4039.

3. Dietetics and Clinical Nutrition Laboratory - Department of Public Health, Experimental and Forensic Medicine. NUTRITIONAL INDICATIONS FOR PATIENTS WITH COVID-19.

4. Cascella M, Rajnik M, Cuomo A, et al. Features, Evaluation and Treatment Coronavirus (COVID-19) [Updated 2020 Mar 8]. In: StatPearls [Internet]. Treasure Island (FL): StatPearls Publishing; 2020 Jan.

5. Dushianthan A, Grocott MP, Postle AD, Cusack R. Acute respiratory distress syndrome and acute lung injury. Postgraduate Medical Journal 2011;87(1031):612-22.

6. P. Singer et al. ESPEN guideline on clinical nutrition in the intensive care unit. Clin Nutr. 2019 Feb;38(1):48-79.

7. Jin YH, Cai L, Cheng ZS, et al. A rapid advice guideline for the diagnosis and treatment of 2019 novel coronavirus (2019-nCoV) infected pneumonia (standard version). Mil Med Res . 2020;7(1):4. Published 2020 Feb 6. doi:10.1186/s40779-020-0233-6.

8. Singer $\mathrm{P}$ et al ESPEN guideline on clinical nutrition in the intensive care unit. Clin Nutr. 2019 Feb;38(1):48-79. Doi: 10.1016/j.clnu.2018.08.037. Epub 2018 Sep 29.

9. SINPE guidelines for Hospital Artificial Nutrition 2002 - Special Part B. Artificial Nutrition in respiratory failure.

10. Roberto Aquilani et al. Correlation of deglutition in subacute ischemic stroke patients with peripheral blood adaptive immunity: Essential amino acid improvement. International Journal of Immunopathology and Pharmacology 28(4).

11. Roberto Aquilani et al. Unaffected Arm Muscle Hypercatabolism in Dysphagic Subacute Stroke Patients: The Effects of Essential Amino Acid Supplementation. BioMed Research International Volume 2014, Article ID 964365, 17 pages.

12. Sebastiano B. Solerte et al. Nutritional Supplements with Oral Amino Acid Mixtures Increases WholeBody Lean Mass and Insulin Sensitivity in Elderly Subjects with Sarcopenia. The American Journal of Cardiology (www.AJConline.org) Vol 101 (11A) June 2, 2008.

13. Ilaria Buondonno et al. From mitochondria to healthy aging: The role of branched-chain amino acids treatment: MATeR a randomized study. Clinical Nutrition, https://doi.org/10.1016/j.clnu.2019.10.013.

14. Saki Inoue \& Hideki Ikeda. Differences in plasma amino acid levels in patients with and without bacterial infection during the early stage of acute exacerbation of COPD. International Journal of COPD 2019:14 575-583.

15. Xiaoshi Ma et al. Protective Effects of Functional Amino Acids on Apoptosis, Inflammatory Response, and Pulmonary Fibrosis in Lipopolysaccharide-Challenged Mice. J. Agric. Food Chem. 2019, 67, 4915-4922.

16. Gisele P. Oliveira et al. Exogenous Glutamine in Respiratory Diseases: Myth or Reality? Nutrients 2016, 8, 76; doi:10.3390/nu8020076.

17. Gisele P. Oliveira et al. Understanding the mechanisms of glutamine action in critically ill patient. 
Anais da Academia Brasileira de Ciências (2010) 82(2): 417-430.

18. Raymond Chang et al. Lactoferrin as potential preventative and treatment for COVID-19. 